\title{
Is the solar spectrum latitude-dependent?
}

\section{An investigation with SST/TRIPPEL}

\author{
D. Kiselman ${ }^{1,2}$, T. M. D. Pereira ${ }^{3, \star}$, B. Gustafsson ${ }^{4,5}$, M. Asplund ${ }^{6,3}$, J. Meléndez ${ }^{7}$, and K. Langhans ${ }^{1,2, \star \star}$ \\ 1 The Institute for Solar Physics of the Royal Swedish Academy of Sciences, AlbaNova University Centre, 10691 Stockholm, \\ Sweden \\ e-mail: dan@astro.su.se \\ 2 Stockholm Observatory, Department of Astronomy, Stockholm University, AlbaNova University Centre, 10691 Stockholm, \\ Sweden \\ 3 Research School of Astronomy and Astrophysics, Australian National University, Cotter Rd., Weston, ACT 2611, Australia \\ ${ }^{4}$ Department of Physics and Astronomy, Uppsala University, Box 515, 75120 Uppsala, Sweden \\ 5 Nordita, AlbaNova University Centre, 10691 Stockholm, Sweden \\ ${ }^{6}$ Max-Planck-Institut für Astrophysik, Postfach 1317, 85741 Garching b. München, Germany \\ 7 Departamento de Astronomia do IAG/USP, Universidade de São Paulo, Rua Matão 1226, São Paulo, 05508-900, SP, Brazil
}

Received 23 June 2011 / Accepted 23 August 2011

\begin{abstract}
Context. In studies of the solar spectrum compared to spectra of solar twin stars, it has been found that the chemical composition of the Sun seems to depart systematically from those of the twins. One possible explanation could be that the effect is caused by the special aspect angle of the Sun when observed from Earth compared with the aspect angles of the twins. This means that a latitude dependence of the solar spectrum, even with the heliocentric angle constant, could lead to the observed effects.

Aims. We explore a possible variation in the strength of certain spectral lines that are used in the comparisons between the composition of the Sun and the twins at loci on the solar disk with different latitudes but at constant heliocentric angle.

Methods. We use the TRIPPEL spectrograph at the Swedish 1-m Solar Telescope on La Palma to record spectra in five spectral regions to compare different locations on the solar disk at a heliocentric angle of $45^{\circ}$. Equivalent widths and other parameters are measured for fifteen different lines representing nine atomic species. Spectra acquired at different times are used in averaging the line parameters for each line and observing position.

Results. The relative variations in equivalent widths at the equator and at solar latitude $\sim 45^{\circ}$ are found to be less than $1.5 \%$ for all spectral lines studied. Translated into elemental abundances as they would be measured from a terrestrial and a hypothetical pole-on observer, the difference is estimated to be within 0.005 dex in all cases.

Conclusions. It is very unlikely that latitude effects could cause the reported abundance difference between the Sun and the solar twins. The accuracy obtainable in measurements of small differences in spectral line strengths between different solar disk positions is very high, and can be exploited in studies of, e.g. weak magnetic fields or effects of solar activity on atmospheric structure.
\end{abstract}

Key words. stars: abundances - instrumentation: spectrographs - line: formation - Sun: abundances

\section{Introduction}

In recent studies it has been found that the chemical composition of the Sun, as compared with those of very similar stars, so-called solar twins, is systematically different: the Sun is comparatively poorer in refractories, i.e. elements that easily condensed to solids for instance in the solar protoplanetary nebula, than in volatiles (Meléndez et al. 2009; Ramírez et al. 2009). The range of the effect is about $20 \%$ for volatiles as compared with refractories. Several possible explanations for this interesting phenomenon have been discussed (see Meléndez et al. 2009; Gustafsson et al. 2010), including cleansing of the protoplanetary disk by planetesimal formation. A more mundane explanation could be an obvious selection effect: in Earth-based studies the Sun is by necessity observed from a position close to the

* Current Address: NASA ARC, Mail Stop 245-3, PO Box 1, Moffett Field, CA 94035, USA.

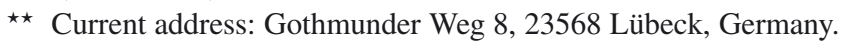

solar equatorial plane. The solar twins, on the other hand, are presumably observed with aspect angles randomly distributed with respect to their rotation axes. Thus, if the solar spectrum, integrated across the disk, for some reason were somewhat different as seen from a position far from the equatorial plane, the differences interpreted as abundance differences might have this explanation instead. An empirical way of exploring this is to observe solar photospheric spectra at different disk positions, though with a common angular distance from the disk centre so that the first-order centre-to-limb variations can be directly compensated for. Here, such a study is presented.

Studies of centre-to-limb variation of the solar spectrum have been pursued for more than a century (cf. Hastings 1873; Hale \& Adams 1907). Some observational studies have been devoted to the question whether there are any differences between spectra from regions along the solar equator as compared with along a meridian. These studies have mostly been concentrated towards variations in line shifts and line shapes (bisectors) 
(e.g., Caccin et al. 1976, 1978; Beckers \& Taylor 1980; Brandt \& Schroeter 1982; Andersen 1984), variations that have generally been interpreted as variations in the photospheric velocity fields with latitude. There are few studies of the variations, or constancy, in the line strengths (equivalent widths). One notable example is the study by Rodríguez Hidalgo et al. (1994) of the variation along the equator and a meridian of four lines of $\mathrm{C}$, $\mathrm{Si}$ I, Mn I, and Fe I. In addition to studies of line widths and bisectors, these authors also included measurements of the variations of the equivalent widths. For Si I, Mn I and Fe I lines they found the lines to be a few percent weaker along the equator than along the meridian at given $\mu \approx 0.6$, while the $\mathrm{C}_{\text {I }}$ line showed less variation. Although not very pronounced, this tendency has the direction needed to explain the apparently low ratio of refractories/volatiles in the Sun compared to the twins. However, three of the four spectral lines studied by Rodríguez Hidalgo et al. are on the flat part of the curve of growth, while the lines used in the analyses of Meléndez et al. (2009) and Ramírez et al. (2009) are almost all on the linear part with line strengths insensitive to the velocity fields (among the lines of Meléndez et al. is C I $538.0322 \mathrm{~nm}$, which is the fourth line measured by Rodríguez Hidalgo et al.). Therefore, the qualitative tendency found by Rodríguez Hidalgo et al. cannot be used to infer what could be the effects on abundances derived from solar flux spectra at different aspect angles. Instead, a direct comparison of line strengths of the mostly weak lines used in the Sun-twin abundance comparison is needed.

It is clear from the previous work on the latitude dependence of the solar spectrum that considerable care must be exercised in positioning the spectrometer slit and averaging properly over granulation and other inhomogeneities. A strictly differential approach must be adopted to minimise the effects of stray light and other instrumental problems.

\section{Observations}

The observations were made with the Swedish 1-m Solar Telescope (SST) on La Palma (Scharmer et al. 2003) employing the TRIPPEL spectrograph during the period of 3-15 May 2010. Because this instrument has not yet been subject to a detailed description in the literature, we use this opportunity to give a summary of its properties.

\subsection{The TRIPPEL spectrograph}

The TRI-Port Polarimetric Echelle-Littrow (TRIPPEL) spectrograph had the following design goals:

- allow simultaneous observations at three separate wavelengths;

- in principle exploit the full spatial resolution of the SST;

- wavelength range about $380 \mathrm{~nm}-1100 \mathrm{~nm}$;

- good polarimetric properties;

- moderate (for a solar spectrometer) spectral resolution $(R \approx$ $200000)$.

To achieve this, it is designed as a lens Littrow spectrograph with a ruled echelle grating. The design draws on the experience from the short Littrow spectrograph at the SVST (Scharmer et al. 1985 , the predecessor of the SST). Some relevant numbers are given in Table 1 and a schematic view of the main optical design is shown in Fig. 1.

The slit is etched onto a chromium-coated glass plate that is easily interchangeable and the reimaging lens of the SST could
Table 1. Some properties of the TRIPPEL spectrograph

\begin{tabular}{lc}
\hline \hline Slit width & $25 \mu \mathrm{m}\left(\approx 0^{\prime \prime} 11\right)$ \\
Grating constant & $79.01 \mathrm{~mm}^{-1}$ \\
Blaze angle & $63.43^{\circ}$ \\
Grating size & $154 \times 306 \mathrm{~mm}$ \\
Focal length & $1500 \mathrm{~mm}$ \\
Littrow lens aperture & $135 \mathrm{~mm}$ \\
\hline
\end{tabular}

also in principle be changed to give another image scale. The spectrograph optics and the grating can accommodate a beam that is twice as fast as was used in the current setup $(f / 46)$. The slit plate can be rotated around a vertical axis to allow the reflected light to be directed towards slit-jaw imagers. It can also be rotated around an horizontal axis orthogonal to the optical axis to adjust the tilt of the slit relative to the dispersion direction. There are two movable jaws behind the slit plate that can be used to cover parts of the slit to minimise the amount of light entering the spectrograph, and to allow dark areas on the spectrum camera for reference.

The Littrow doublet-lens is mounted on a translational stage. To obtain the required large diffraction-limited field-of-view, a meniscus field lens is placed close to the focal plane so that the light passes twice through it. The optical design fixes the Littrow lens position relative to this field lens. The translational stage is thus only used when checking the spectrograph focus and never for focusing the spectrograph cameras.

The grating from Richardson Grating Laboratory has excellent polarization properties, with the maximum difference between $\mathrm{S}$ and $\mathrm{P}$ efficiency being about $10 \%$ in the visual region. The grating is mounted on a rotational stage with $0.01^{\circ}$ setting precision.

Three silver-coated mirrors pick off light to the exit ports. For the two ports with horizontal light beams (designated A and B), the cameras are mounted on holders sliding on a rail of the same type as is used for the SST imaging setups, thus allowing freedom in camera choice. The third port (C), which has a vertical light beam, currently uses a special holder for a Redlake Megaplus II camera (ES1603).

Apart from some image distortion caused by the corrector lens, TRIPPEL has an off-plane design that leads to curvature of the spectral lines (smile) and also of the dispersion direction (keystone). The curvature over the typical CCD size is small, so the greatest effect of the smile is to cause the spectral lines to tilt relative to the axis defined by the direction of the grating grooves. The slit is rotated about $3.5^{\circ}$ from the vertical direction to partly compensate for this.

Because all spectral orders fall on top of each other in the focal plane, interference filters placed just in front of the CCDs are used to select the desired order. This also has the advantage of blocking straylight outside the filter passband. The transmission profile of two-cavity filters typically has a base that is too wide to be practical, so three-cavity filters are used. Assuming a profile for a three-cavity interference filter, requiring that the transmission at a distance of a full spectral range from the central wavelength is $10^{-4}$ of its peak value, and applying a significant margin of safety, results in the filter widths in Table 2.

The dispersion decreases with decreasing order and thus with increasing wavelength. The theoretical spectral resolution is limited by the slit width and so stays fairly constant between different orders: it is between 1.1 and $1.4 \mathrm{~km} \mathrm{~s}^{-1}$ or $R=\frac{\lambda}{\delta \lambda} \sim$ 220000 . The resolution as found by our straylight-fitting routine is generally lower than this, but there is probably cross-talk 
Table 2. Nominal TRIPPEL parameters at the wavelengths of some interesting lines.

\begin{tabular}{lcccccc}
\hline \hline Line & $\begin{array}{c}\lambda \\
{[\mathrm{nm}]}\end{array}$ & $\begin{array}{c}F W H M \\
{[\mathrm{~nm}]}\end{array}$ & $n$ & $\begin{array}{c}\text { Bandpass } \\
{\left[\mathrm{km} \mathrm{s}^{-1}\right]}\end{array}$ & $\begin{array}{c}\mathrm{d} x / \mathrm{d} \lambda \\
{[\mathrm{mm} / \mathrm{nm}]}\end{array}$ & $\begin{array}{c}W \\
{[\mathrm{~nm}]}\end{array}$ \\
\hline $\mathrm{Ca}_{\text {II }}$ & 393.39 & 2 & 57 & 1.3 & 14.9 & 0.93 \\
$\mathrm{Mg}_{\mathrm{I}}$ & 457.11 & 2.5 & 49 & 1.3 & 13.0 & 1.06 \\
$\mathrm{Mg}_{\mathrm{I}}$ & 517.27 & 3 & 44 & 1.2 & 11.9 & 1.16 \\
$\mathrm{Fe}_{\mathrm{I}}$ & 630.20 & 5 & 36 & 1.2 & 9.6 & 1.44 \\
$\mathrm{Li}_{\text {I }}$ & 670.78 & 6 & 34 & 1.2 & 9.3 & 1.49 \\
$\mathrm{Ca}_{\text {II }}$ & 866.20 & 10 & 26 & 1.3 & 6.7 & 2.05 \\
\hline
\end{tabular}

Notes. FWHM: recommended width of a three-cavity interference filter. $n$ : spectral order. Bandpass: slit-limited. $\mathrm{d} x / \mathrm{d} \lambda$ : dispersion. $W$ : wavelength coverage for a $13.8 \mathrm{~mm} \mathrm{CCD} \mathrm{(Megaplus} \mathrm{1.6).}$
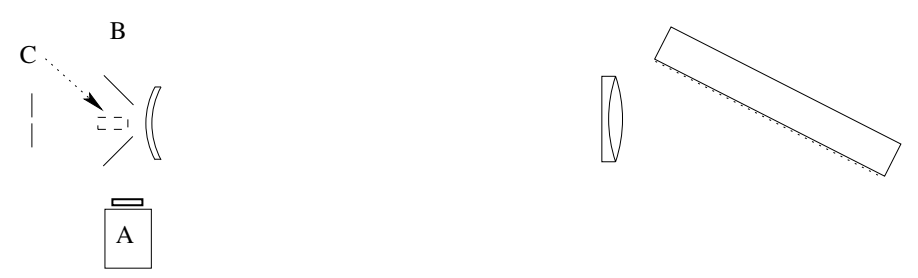

Fig. 1. Schematic view of the TRIPPEL spectrograph as seen from above. Components from left to right: entrance slit, pick-off mirrors for ports A-C, field lens, Littrow doublet, grating. Spectral port A has a CCD camera with an interference filter.

between the spectral resolution and the straylight parameter in our model.

The spectral interval recorded by each camera is determined by the turning angle of the grating and the filter. With three ports, many combinations are possible, especially because some freedom of camera movement in the dispersion direction is available.

Table 2 lists some nominal instrumental parameters at the wavelengths of some interesting lines.

The spectrograph is focused by pressing a perpendicular slit against the spectrograph slit. This creates a small rectangle that is seen on the spectrum cameras as a very narrow spectrum. The Littrow lens is then moved between two out-of-focus position for a range of camera positions. The camera position where the two lens positions create spectra of similar sharpness is taken as its focus position. With some training it is not difficult to assess the sharpness from the spectrum image displayed on the screen.

The other crucial focusing, that of the solar image on the spectrograph slit, is done with a similar technique. But instead of placing a physical slit crossing the spectrograph slit, an image of a slit is placed there by mounting a physical slit in the telescope focus at the exit port of the vacuum system. The adaptive optics is locked on a pinhole next to that slit. The wavefront sensor is then adjusted (which leads to the image moving back and forth) until symmetry in the out-of-focus spectra is achieved.

Finally, TRIPPEL allows some auxiliary instruments. The wavefront sensor that controls the adaptive mirror and the correlation tracker camera are fed via a beamsplitter cube in front of the slit. The reflective slit plate makes slit-jaw imaging possible. For the current observations, we employed up to two slitjaw cameras fed with reimaging optics. These cameras were equipped with interference filters with central wavelengths close to the spectral regions observed. The slit-jaw imaging was used for pointing control, and for keeping track of the seeing quality and the light-intensity level.
Table 3. Spectral lines observed.

\begin{tabular}{rlrrl}
\hline $\begin{array}{r}\text { Spectral } \\
\text { region }\end{array}$ & Species & $\begin{array}{r}\text { CWL } \\
{[\mathrm{nm}]}\end{array}$ & $\begin{array}{r}E_{l} \\
{[\mathrm{eV}]}\end{array}$ & Class \\
\hline 538 & $\mathrm{C}_{\mathrm{I}}$ & 538.03 & 7.58 & volatile \\
616 & $\mathrm{Na}_{\mathrm{I}}$ & 616.08 & 2.10 & intermediate \\
616 & $\mathrm{Fe}_{\mathrm{I}}$ & 616.54 & 4.14 & refractory \\
616 & $\mathrm{Ca}_{\mathrm{I}}$ & 616.64 & 2.52 & refractory \\
616 & $\mathrm{Ca}_{\mathrm{I}}$ & 616.90 & 2.52 & refractory \\
711 & $\mathrm{C}_{\mathrm{I}}$ & 711.14 & 8.64 & volatile \\
711 & $\mathrm{C}_{\mathrm{I}}$ & 711.32 & 8.65 & volatile \\
768 & $\mathrm{~S}_{\mathrm{I}}$ & 768.61 & 7.87 & intermediate \\
768 & $\mathrm{~K}_{\mathrm{I}}$ & 769.90 & 0.00 & intermediate \\
777 & $\mathrm{O}_{\mathrm{I}}$ & 777.19 & 9.15 & volatile \\
777 & $\mathrm{O}_{\mathrm{I}}$ & 777.41 & 9.15 & volatile \\
777 & $\mathrm{O}_{\mathrm{I}}$ & 777.53 & 9,15 & volatile \\
783 & $\mathrm{Al}_{\mathrm{I}}$ & 783.53 & 4.02 & refractory \\
783 & $\mathrm{Al}_{\mathrm{I}}$ & 783.61 & 4.02 & refractory \\
869 & $\mathrm{~S}_{\mathrm{I}}$ & 869.46 & 7.87 & intermediate \\
\hline
\end{tabular}

\subsection{Spectral lines}

In the present study we have selected some spectral lines used by Meléndez et al. (2009) in their analysis of the Sun and solar twins, ensuring that both refractories, volatiles, and intermediate elements were represented and that as many lines as possible were observable simultaneously. The spectral regions were ranked in priority and the setup was changed to the next set of regions when enough data had been secured. Table 3 lists the lines observed and Table 4 the setup combinations used. Figure 4 shows plots of all the lines in their spectral context of our solar observations.

\subsection{Observations: strategy and practice}

We assume that a latitude dependence of line equivalent widths that is strong enough to give a significant effect in the flux spectrum must be present in the intensity spectrum at a moderate distance from solar disk centre. We chose a heliocentric distance of 45 degrees or $\mu=\sqrt{2} / 2 \sim 0.71$. For the solar meridian, this corresponds to the point that is seen at the same $\mu$ for an observer in the ecliptic plane and one that would observe the Sun pole-on. It is also close to $\mu=2 / 3$, which is where, according to the Eddington-Barbier relations, the outgoing intensity spectrum should be characteristic of the flux spectrum and thus relevant for stellar observations.

In order to decrease bias from north-south and east-west systematic differences that are perhaps caused by random activity patterns, we selected eight different position angles and named these points N, W, S, E, NW, SW, SE, and NE. To test the stability of the results, the solar disk centre was observed frequently and interspersed with the $\mu=0.71$ pointings. The solar latitudes of the observed points are given in Table 4 . The heliographic latitude at the time of observations was approximately $-3^{\circ}$.

An observational sequence consisted of a series of pointings with about 5 min spent at each. Before and after each sequence flat fields were acquired. The sequence typically started at disk centre and then went to all eight $\mu=0.71$ points, after which the disk centre was observed again. During the observations the $\mathrm{Ca}$ II $\mathrm{H}$ imaging camera was used constantly to ensure that the regions observed were quiet.

After the first days of observations, when an inspection of the data confirmed that the stability of the results was quite high, the intermediate points (NW, SW, SE, NE) were abolished from 
A\&A 535, A14 (2011)

Table 4. Observational setups and the number of data sets from each day used in the final analysis.

\begin{tabular}{lcccccccccccc}
\hline \hline Date & Port A & Port C & Port B & $\begin{array}{c}\text { DC } \\
-3^{\circ}\end{array}$ & $\begin{array}{c}\text { N } \\
42^{\circ}\end{array}$ & $\begin{array}{c}\text { NW } \\
27^{\circ}\end{array}$ & $\begin{array}{c}\text { W } \\
-3^{\circ}\end{array}$ & $\begin{array}{c}\text { SW } \\
-32^{\circ}\end{array}$ & $\begin{array}{c}\text { S } \\
-48^{\circ}\end{array}$ & $\begin{array}{c}\text { SE } \\
-32^{\circ}\end{array}$ & $\begin{array}{c}\text { E } \\
-3^{\circ}\end{array}$ & $\begin{array}{c}\text { NE } \\
27^{\circ}\end{array}$ \\
\hline $2010-05-05$ & 538 & 711 & 783 & 5 & 1 & 1 & 1 & 1 & 1 & 1 & 0 & 1 \\
$2010-05-06$ & 538 & 711 & 783 & 9 & 3 & 3 & 3 & 3 & 3 & 3 & 3 & 2 \\
$2010-05-07$ & 538 & 711 & 783 & 7 & 3 & 1 & 3 & 1 & 3 & 1 & 3 & 1 \\
$2010-05-09$ & 768 & $\ldots$ & 777 & 6 & 5 & 0 & 4 & 0 & 5 & 0 & 5 & 0 \\
$2010-05-14$ & 768 & $\ldots$ & 777 & 1 & 1 & 0 & 0 & 0 & 0 & 0 & 0 & 0 \\
$2010-05-15$ & 869 & $\ldots$ & 616 & 7 & 4 & 0 & 4 & 0 & 4 & 0 & 4 & 0 \\
\hline
\end{tabular}

Notes. DC is solar disk centre. Solar latitudes are given for each disk position.

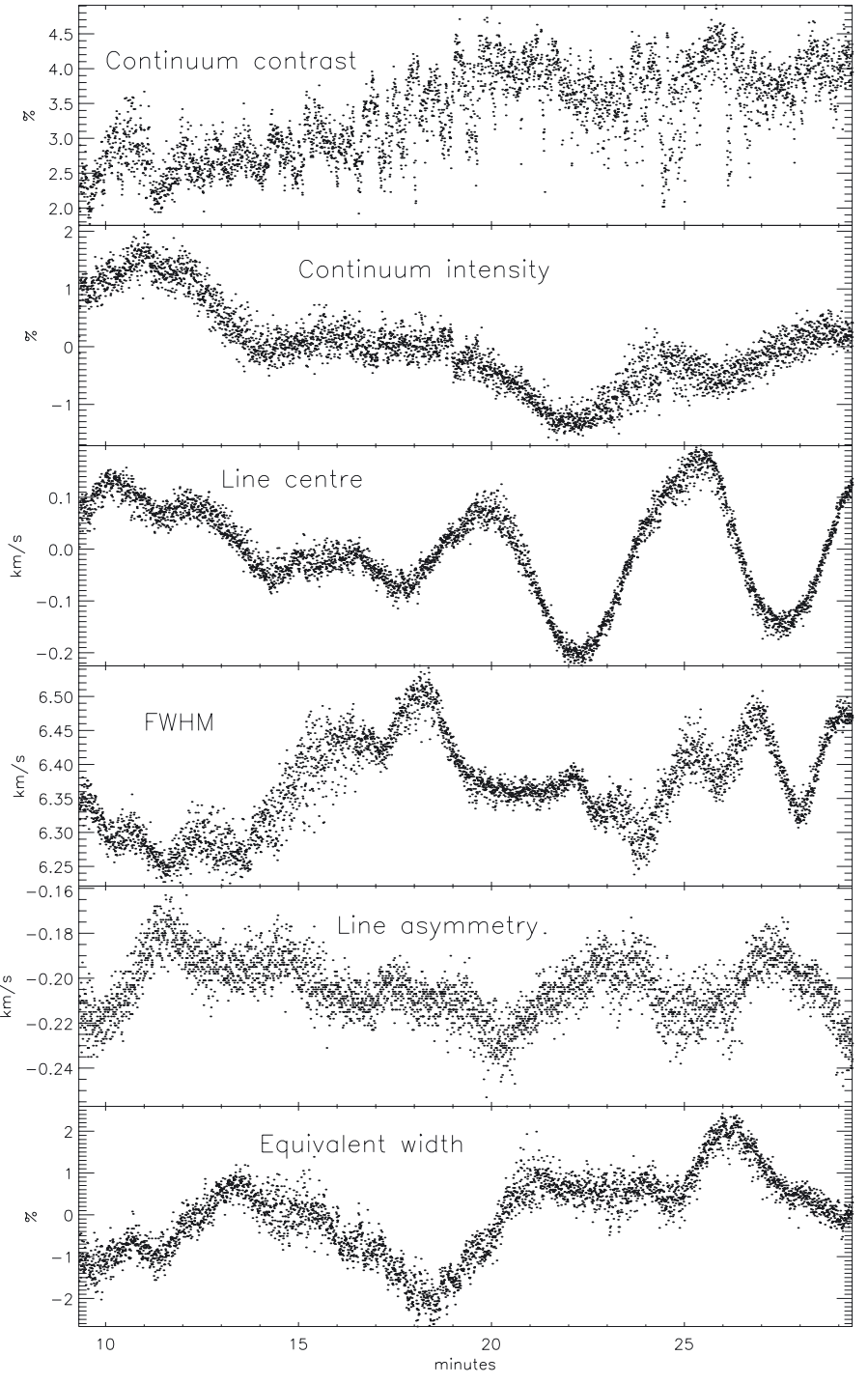

Fig. 2. Temporal sequence of the $\mathrm{Al}_{\mathrm{I}} 783 \mathrm{~nm}$ line at disk center. The six quantities are plotted as a function of time in minutes after 10:00 UT. Equivalent width and continuum intensity are plotted as the deviation in percent from their respective mean values. Each point corresponds to the mean spectrum along the slit for one exposure.

the observing sequence, and only observations in the $\mathrm{N}, \mathrm{W}, \mathrm{S}$, and $\mathrm{E}$ positions as well as the disk centre were made.

The first data sets were taken with fixed pointing. In order to increase the spatial sampling, a procedure was implemented where the slit was scanning back and forth over $3^{\prime \prime}$. This was made via the correlation tracker.

\subsection{Data reductions}

The data reduction procedures follow those described by Pereira et al. (2009) with few deviations. Each data set requires a set of flat-field and dark exposures. The flat-fields are acquired by taking many exposures while the telescope is scanning over a designated area around disk centre that should be devoid of spots. For the current observations, a new procedure was used where the adaptive mirror of the SST is fed with random voltages to smear out spatial structures even more. The darks are acquired with the light beam blocked close to the telescope exit window.

The flat-field exposures were used to compute gain tables and map the geometric distortions of the spectrograms. They are also used for wavelength calibration and scattered-light corrections in a procedure similar to that of Allende Prieto et al. (2004), thus comparing the observed spectra with the Fourier Transform Spectrometer at the McMath-Pierce Telescope (hereafter called the FTS atlas) of Brault \& Neckel (1987).

Wavelength calibration was performed by fitting line cores. The resulting wavelength scale is thus that of the FTS atlas.

Straylight inside the spectrograph was treated as a constant that is determined simultaneously with the spectral resolution by a least-squares fit to the reference spectrum. Typical values are $5-6 \%$ of the spectral continuum.

Telluric lines were identified and excluded from the fitting procedures.

Remaining artefacts in the continuum level - caused for example by residual components of fringes perpendicular to the dispersion direction - were also removed with the help of the reference spectrum. The result is a set of parameters that maps the observed disk-centre average spectrum to that of the atlas.

The process described above produces spectrograms ready for analysis. In the current work, we proceeded by coadding each spectrogram over the spatial direction to obtain a onedimensional spectrum that is the average spectrum under the spectrograph slit. A number of these spectra are then coadded, thus forming an average also over time. Various quantities for the spectral lines of interest were then calculated from these average spectra: line centre is the velocity difference between the local line core and the calibration spectra - it is thus relative to the FTS atlas; FWHM is the usual full-width-at-half-maximum line depth; line asymmetry is the distance between the local line core and the line bisector at half-maximum line depth; equivalent widths were measured by direct integration over a predefined interval that is centred on the line core and thus follows any local Doppler shift - this is illustrated in Fig. 4. 
D. Kiselman et al.: Is the solar spectrum latitude dependent?
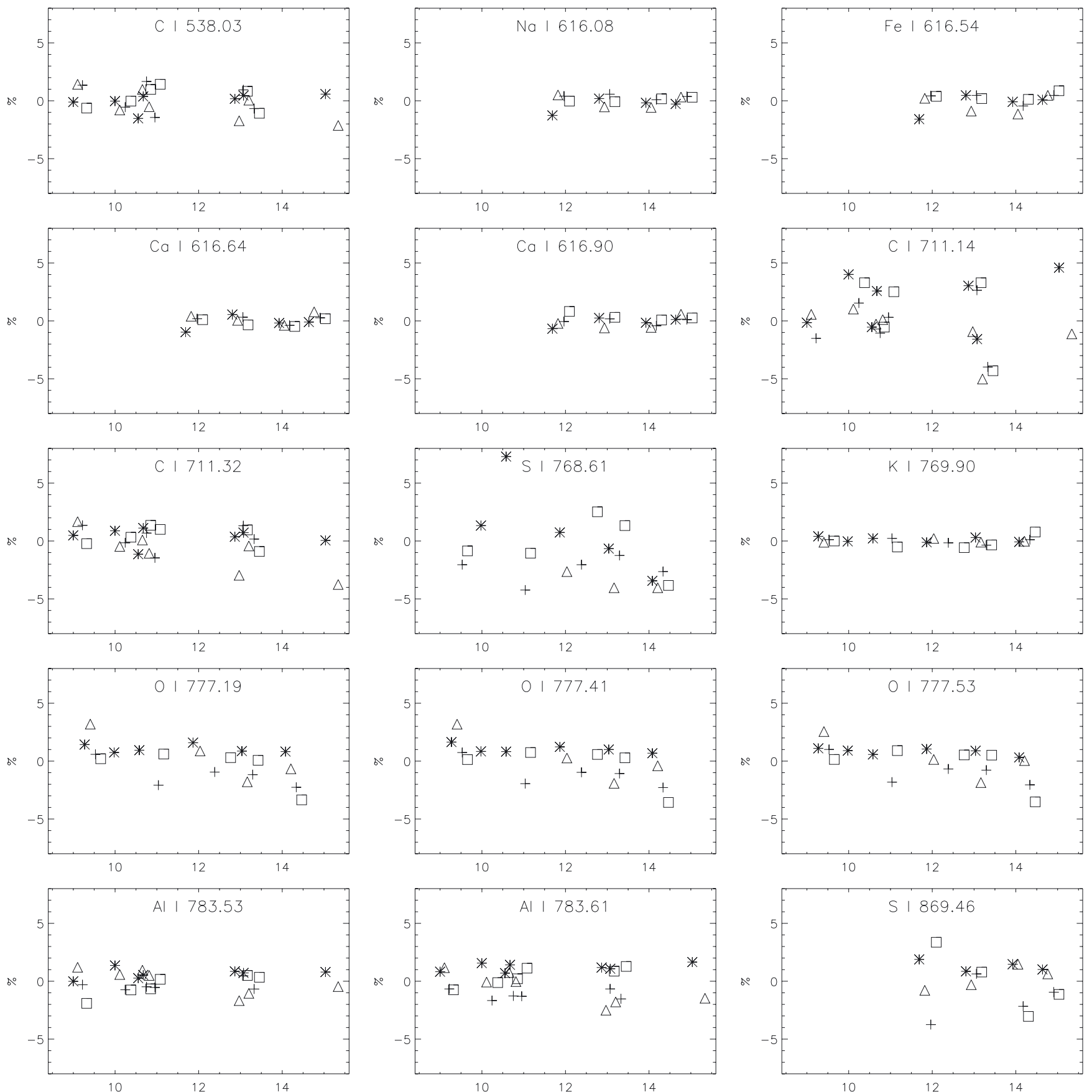

Fig. 3. Equivalent widths for all lines and data sets for $\mu=\sqrt{2} / 2$. The deviation from the mean value from each data set is plotted in units of percent of the overall mean value and as a function of the time of day in hours when it was acquired. Symbols according to solar disk position. Asterisk: N, plus: S, triangle: W, square: E.

\subsection{Data selection and error discussion}

Sources of errors and other effects that would undermine the observational strategy could be imprecise and non-reproduceable pointing, seeing, variations on the Sun from granulation, oscillations, or activity, and instrumental drifts. Figures 5-7 illustrate this by showing several disk-centre spectral profiles of three lines.

\subsubsection{Pointing}

The exact pointing of the telescope is of utmost importance because the equivalent width of the most sensitive lines in our sample - the $C_{\text {I }}$ lines at $711 \mathrm{~nm}$ - is predicted to change by $5 \%$ for a $1^{\prime}$ shift $(\Delta \mu \approx 0.05)$ along the radius vector. We used the solar limb in four positions to calibrate the telescope pointing before each sequence. After such a sequence we returned to the solar limb to estimate the drift in pointing during the sequence. Sometimes this error was found to be small but it could typically amount to $25^{\prime \prime}$.

The spectrograph slit is fixed in the laboratory frame and its orientation in the sky plane will change during the day. No effort was made to measure the orientation. The finite slit length $\left(\sim 50^{\prime \prime}\right)$ thus adds to the positional coverage of each spectrum, but does not affect the mean position of the slit. 

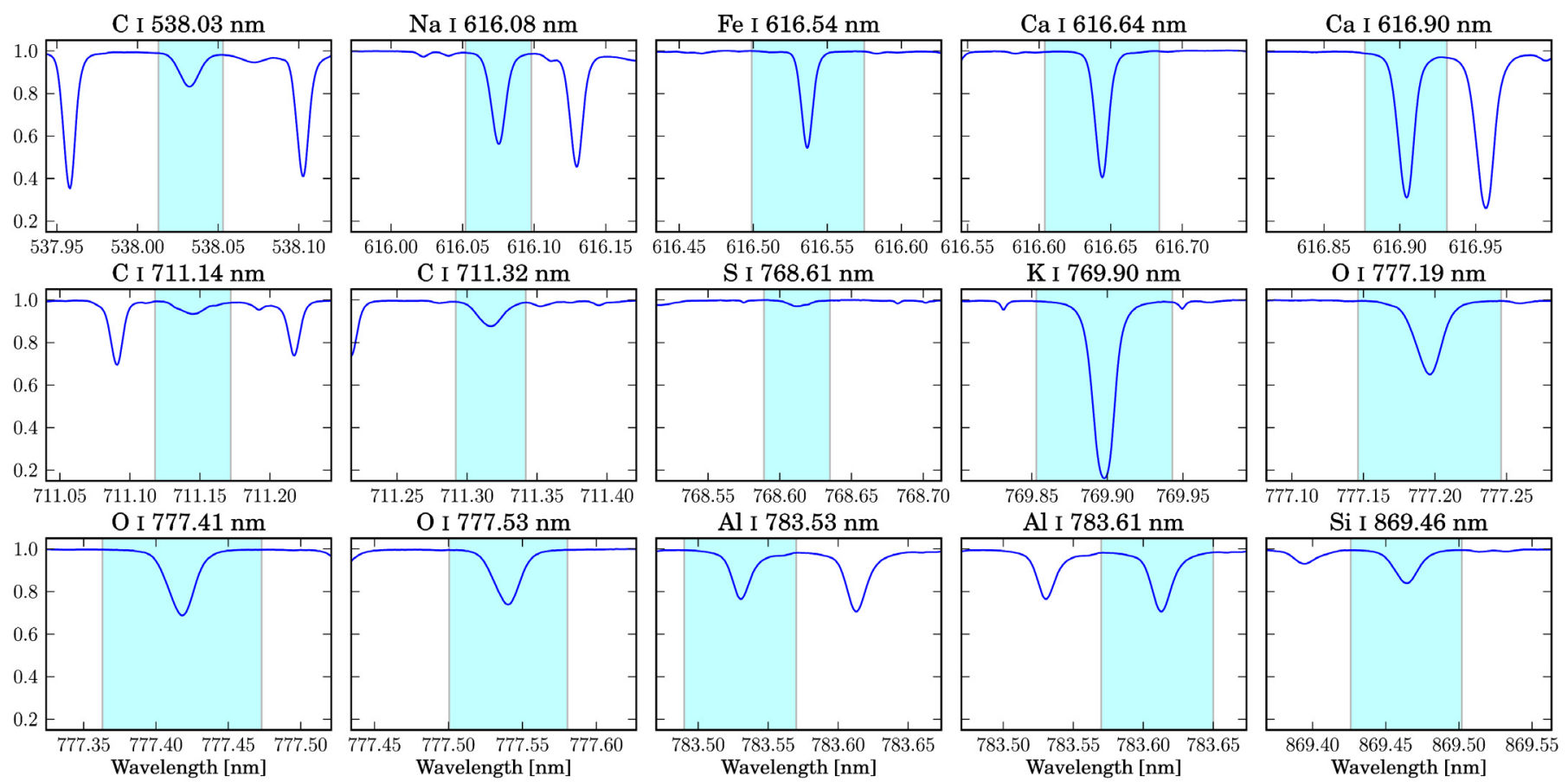

Fig. 4. All spectral lines of the present study. Shaded region shows the integration range for the equivalent widths. This range is shifted according to the line core for every individual spectrum.

\subsubsection{Telluric lines}

If a line is significantly blended with a telluric line, its measured properties will be a function of the airmass and thus the time of the observations. This is obvious for the $\mathrm{C}_{\mathrm{I}} 711.14 \mathrm{~nm}$ line as seen in Fig. 6 (where the standard deviation of $\Delta W / W=2.8 \%$ ), but still this airmass dependence drowns in the noise for this weak line because no clear trend with time is visible in Fig. 3.

\subsubsection{Impact of seeing}

The ever-changing seeing causes the spatial resolution to vary with time. Because the current project does not require very high spatial resolution - indeed some spatial averaging is necessary and beneficial, one might surmise that seeing is irrelevant. We did not take that approach. Bad seeing implies that light from far away on the solar disk will be scattered into the spectrograph slit without any control. We therefore compared results from periods with different seeing but could not discern any significant differences in the measured line properties. Still we excluded the data sets with the worst seeing, and for those that were included, we kept the 50 best frames (100 for disk-centre spectra) as selected from continuum contrast values. We note that really bad seeing can cause tracking loss, which itself is a source of error.

We caution, however, against assuming that seeing is unimportant in studies like this. We note that in our case the observed points were far from the limb, that the centre-to-limb variation of the equivalent widths are fairly linear (thus symmetric blurring will still give the same results), and that the intensity spectrum at this $\mu$ should anyway be similar to the flux spectrum.

A similar comparison of data from periods where thin high clouds were intermittently in view gave a different result. Here the spectra did change significantly and any cloud-affected observations were subsequently excluded from analysis.

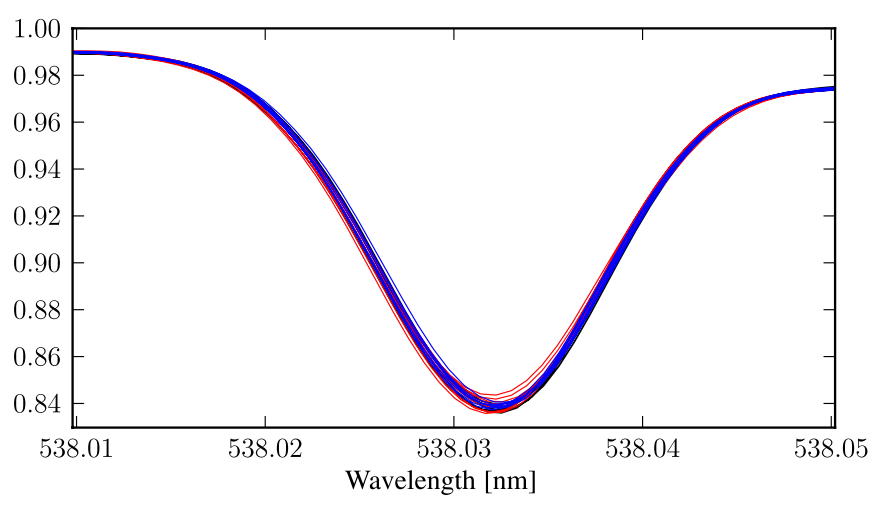

Fig. 5. Disk-centre spectra for $\mathrm{C}_{\mathrm{I}} 538 \mathrm{~nm}$. Black 2010-05-05, Blue 2010-05-06. Red 2010-05-07.

\subsubsection{Solar variations and instrumental stability}

Intrinsic variations of the solar target and instrumental drifts can in principle be difficult to disentangle. Figure 2 shows the result of a run where the telescope was pointed at disk centre for more than $20 \mathrm{~min}$. Each point in the plot corresponds to one exposure. Pointing errors should be negligible here. As seen from the continuum contrast, the seeing was varying but improved during the period. The line-centre variations show clear evidence of 5 -min oscillations. The continuum intensity is clearly correlated with these. The variations of the other line parameters do not seem to correlate with oscillations but seem consistent with being caused by granulation - mainly from its time evolution but probably with contribution from slit drifts and rotation relative to the solar image. There is a noise envelope, probably caused by a combination of photon noise and seeing jitter, totally amounting to about $1 \%$ in equivalent width. 


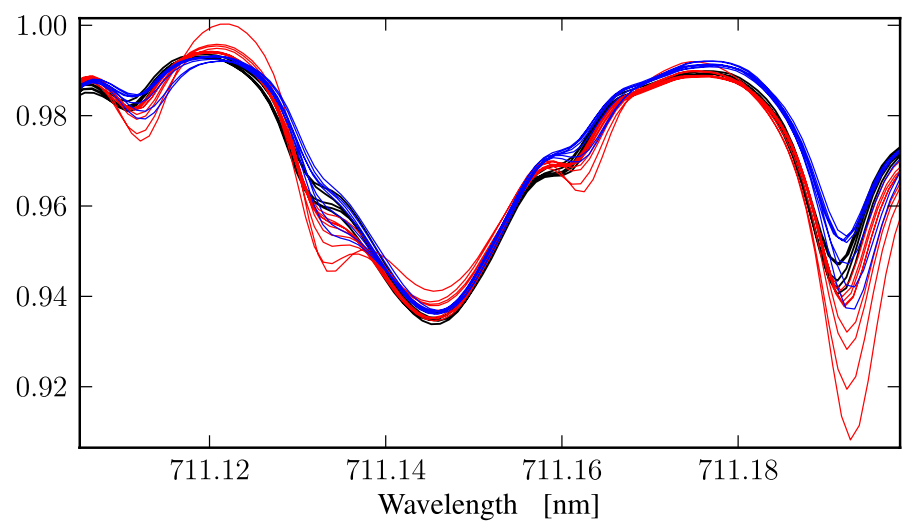

Fig. 6. Like Fig. 5 but for $\mathrm{C}_{\mathrm{I}} 711.14 \mathrm{~nm}$. Note the presence of telluric lines, whose strengths varies with the air mass.

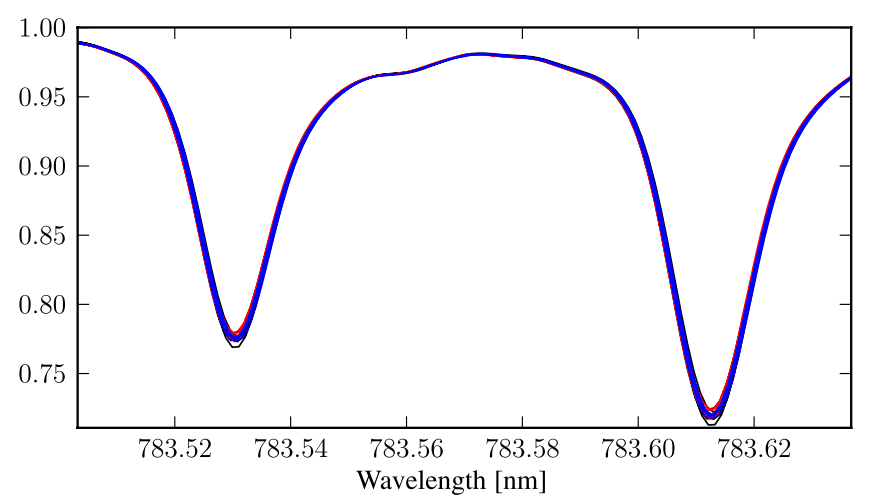

Fig. 7. Like Fig. 5 but for the two lines of $\mathrm{Al}$ I at $783 \mathrm{~nm}$.

\subsubsection{Summary}

All in all, the differential approach taken here should minimise many systematic errors. The results from the tests are consistent with the dominant errors being caused by solar variations and by pointing. These are random in nature, and given the number of data sets, it seems reasonable to use the spread of the individual data points - graphically shown in Fig. 3 - to estimate the uncertainties in the mean values using the standard error of the mean computed separately for the $\mathrm{S}+\mathrm{N}$ data points and the $\mathrm{W}+\mathrm{E}$ datapoints.

\section{Quiet-Sun results}

Figure 3 displays the equivalent widths from all data sets. Different symbols are used for the four different disk positions. From the plots alone, no clear difference between the different disk position is obvious for any line, with one possible exception. We note that for the three $\mathrm{O}$ I lines, four of the $\mathrm{S}$ points lie below all of the $\mathrm{N}$ points. That the fifth point does not, indicates that this N-S divergence may be caused by activity or erroneous pointing because the four deviating data sets were recorded on a different day.

Figure 8 illustrates the data points by showing examples of line profiles for the different disk positions for four lines recorded during the same day.

Computing the mean values separately for the $\mathrm{S}+\mathrm{N}$ and $\mathrm{E}+\mathrm{W}$ disk positions, and using the standard error in each mean value as error estimate, gives the result in Fig. 9. Here, the relative difference in low-latitude (average of $\mathrm{E}$ and $\mathrm{W}$ ) and highlatitude (average of $\mathrm{N}$ and $\mathrm{S}$ ) equivalent width,

$\frac{\Delta W}{\langle W\rangle}=\frac{\left(W_{\text {low }}-W_{\text {high }}\right)}{\langle W\rangle}$,

is plotted for each line. The error bars are the standard errors in the mean values $(1 \sigma)$. The departure from zero of the relative differences $\Delta W /\langle W\rangle$ must be considered insignificant.

Our initial goal was to estimate the difference between the spectral analysis of an observer close to the equatorial plane of the Sun and one who sees the Sun pole on. The polar observer will see a flux spectrum that is characterised by our high-latitude spectra. The equatorial - or terrestrial - observer will see a flux spectrum that is a mix of low-latitude and high-latitude contributions. We approximate this mix to be $1: 1$. Consequently the effective relative difference in flux equivalent width is

$\frac{\Delta W^{\mathrm{eff}}}{\langle W\rangle}=\frac{1}{2} \frac{\Delta W}{\langle W\rangle}$

The final result is shown in Fig. 10 where the difference in derived abundances per element is plotted as a function of condensation temperature. The average values used for this plot were computed without any weights.

The difference in derived abundance was computed with the help of theoretical LTE curves of growth using $\Delta W^{\text {eff }} /\langle W\rangle$ as input. The difference between "polar" and "equatorial" abundances is within 0.005 dex for all elements and to within 0.002 dex for the refractory elements. The error bars are computed directly from those in Fig. 9. Clearly, no significant difference and, in particular, no trend with condensation temperature.

\section{Solar activity}

The principal goal of this work was to look for possible latitudeeffects in the quiet Sun and efforts were made to avoid any activity. The result was negative, but that leaves the possibility that the Sun, or a star, could have significant inclination-dependent spectra caused by activity. The point is that even if two stars have the same level and kind of magnetic activity as the Sun, the active regions tend to keep to lower latitudes. Alternatively, one could argue that the studies by Meléndez et al. (2009) and Ramírez et al. (2009) were made when the Sun was in an unusual deep activity minimum. It the solar twins happened to have systematically higher levels of activity, perhaps their spectra would be different, regardless of inclination angle.

An investigation of this possibility is beyond the scope of this paper. (During our observing run, some data were indeed acquired from regions of moderate activity, but the amount of data is not enough to allow any conclusions.) However, some tentative remarks can be made based on the existing literature.

It seems likely that lines that are strongly affected by activity would also display a variation with the solar cycle. Indeed, the long-term monitoring of solar spectral features of Livingston et al. (2007) shows that some strong lines with chromospheric contributions display variations in phase with the solar cycle. But among weaker, clearly photospheric, lines only Mn I $539.47 \mathrm{~nm}$ shows equivalent-width variations of $2 \%$ during the cycle. The reason why this line is special is explained by Vitas et al. (2009) as caused by its strong hyperfine broadening which leads to a lack-of-broadening-in-granules effect and very high contrast of magnetic structures in the line. Otherwise, C $C_{\text {I }} 538 \mathrm{~nm}$ was found to be constant by Livingston et al. (2007) within $1.5 \%$ $(\Delta \log \epsilon<0.006 \mathrm{dex})$ with no signs of cycles or trends, as were 

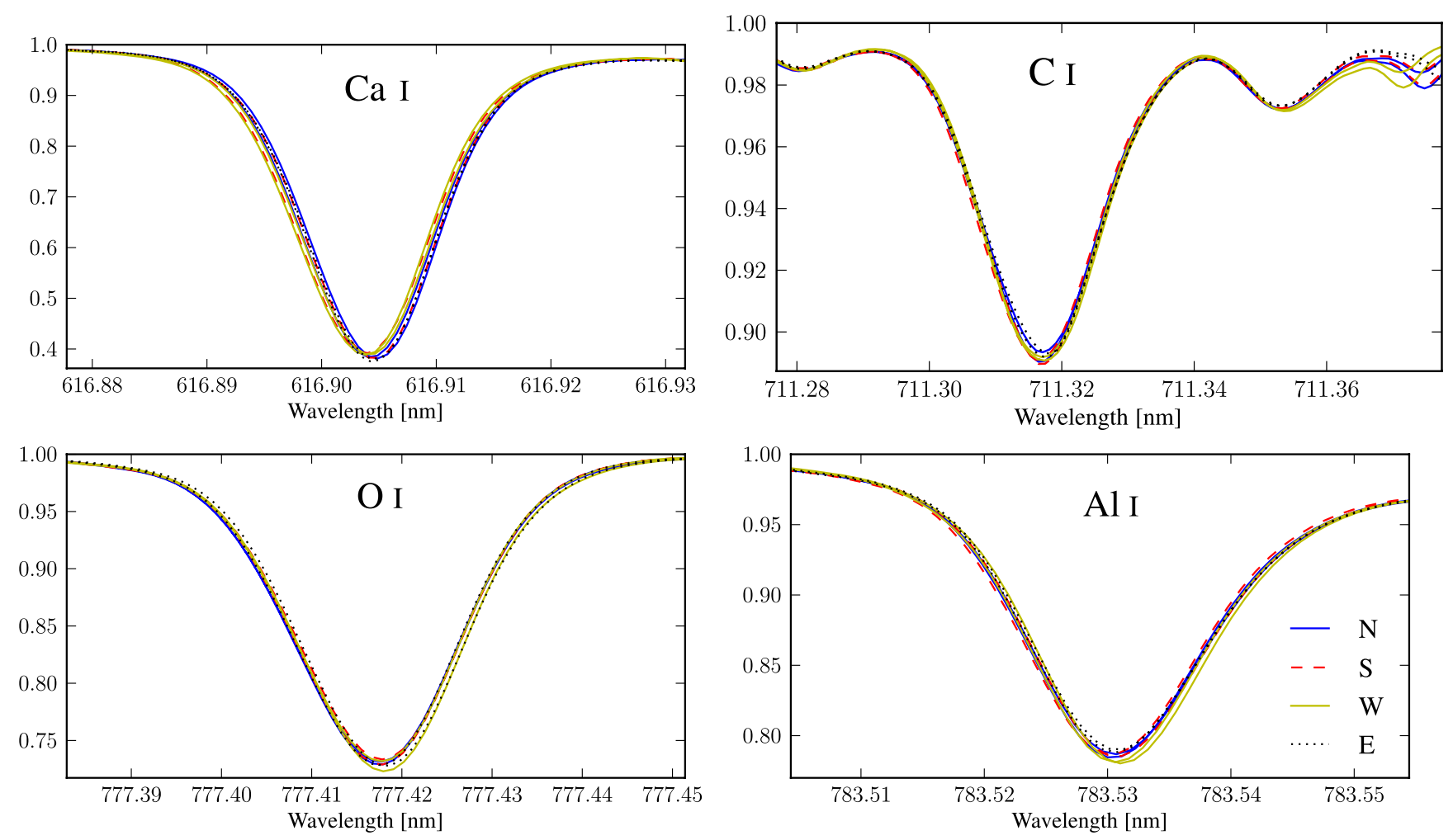

Fig. 8. Two sample spectra for each of the four main disk-positions acquired during the same day. The E and W spectra were adjusted for solar rotation by applying a nominal Doppler shift of $\pm 1.89 \mathrm{~km} \mathrm{~s}^{-1} \sin 45^{\circ}$.

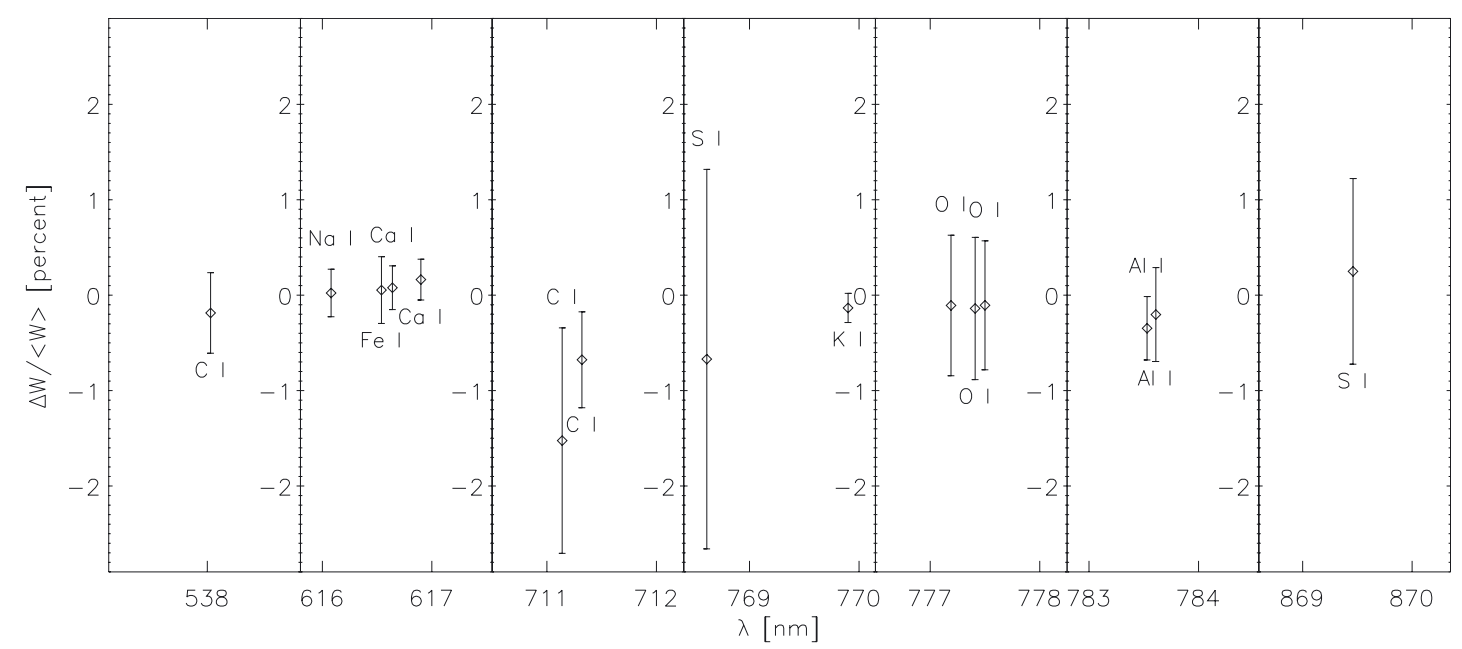

Fig. 9. Difference between low-latitude and high-latitude equivalent widths for all lines. Error bars are based on the standard errors in the respective mean values.

other photospheric lines, e.g., the 777-nm O I triplet, whose equivalent widths all lie within their noise envelope of $1 \%$.

So, while the influence of magnetic activity on lines used for abundance analysis is well worth more investigations, it is unlikely to explain abundance patterns like those found by Meléndez et al. (2009) and Ramírez et al. (2009).

\section{Discussion and conclusions}

We draw the following conclusions:

The TRIPPEL Spectrograph performs well and has a high stability suitable for comparative studies of the kind presented here.
The method of differential comparison is precise and insensitive to, e.g., seeing variations.

The difference in equivalent widths for the lines observed at the equator and at latitudes around $45^{\circ}$ is less than $1 \%$ at $\mu=\sqrt{2} / 2$, except for the telluric-afflicted $C_{\mathrm{I}} 711.1 \mathrm{~nm}$ line. Our results exclude the possibility that the indications of depletion of refractory elements found by Meléndez et al. (2009) in the Sun as compared with the solar twins could be caused by systematically different aspect angles when observing the Sun compared with when observing the twins.

Finally, we note that while we have argued that it is unlikely that solar activity could modify our qualitative conclusions, the effects of magnetic activity on abundances derived 
D. Kiselman et al.: Is the solar spectrum latitude dependent?

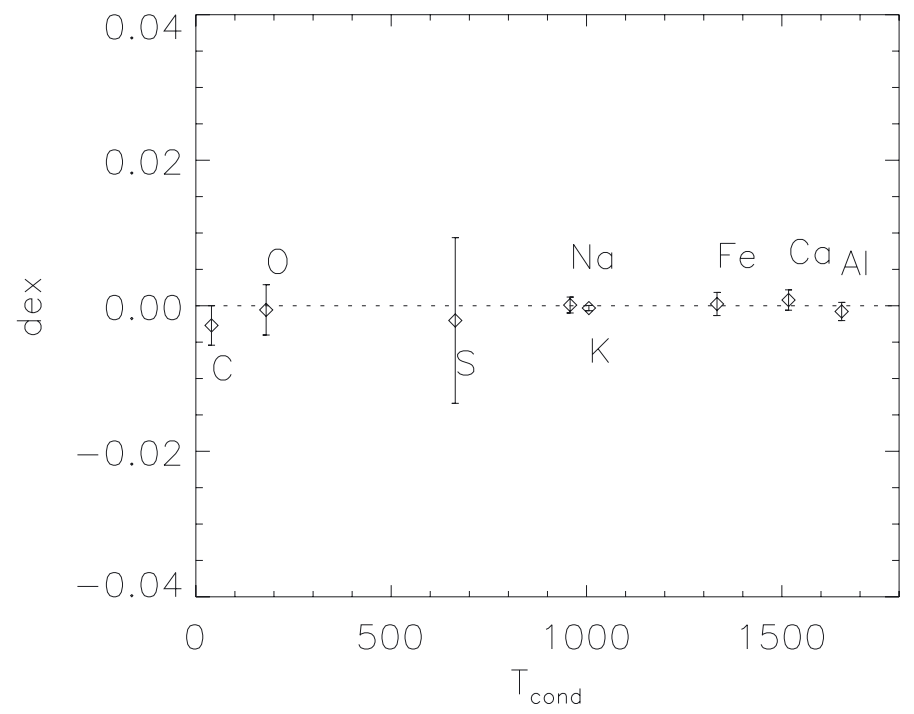

Fig. 10. Difference between "polar" and "equatorial" abundances for all elements as a function of their condensation temperatures according to Lodders (2003).

from high-resolution spectra need further investigation. A natural follow-up study to the current work would be to apply the same methods to explore in detail the differences between photospheric spectra in solar active and inactive regions. In the present age of high-precision spectroscopy, in particular in detailed differential work, it may be important to take the activity state into consideration when one makes detailed abundance analysis.
Acknowledgements. The Swedish 1-m Solar Telescope is operated on the island of La Palma by the Institute for Solar Physics of the Royal Swedish Academy of Sciences in the Spanish Observatorio del Roque de los Muchachos of the Instituto de Astrofísica de Canarias. BG acknowledges support from the Swedish Research Council, VR. J.M. thanks support from FAPESP (2010/17510-3). K.L. was funded by the European Community's Human Potential Program through the European Solar Magnetism Network (contract HPRN-CT-2002-00313). The TRIPPEL spectrograph was built with support from the Swedish Research Council.

\section{References}

Allende Prieto, C., Asplund, M., \& Fabiani Bendicho, P. 2004, A\&A, 423, 1109 Andersen, B. N. 1984, Sol. Phys., 94, 49

Beckers, J. M., \& Taylor, W. R. 1980, Sol. Phys., 68, 41

Brandt, P. N., \& Schroeter, E. H. 1982, Sol. Phys., 79, 3

Brault, J. W., \& Neckel, H. 1987, Spectral Atlas of Solar Absolute Diskaveraged and Disk-Center Intensity from 3290 to $12510 \AA$, ftp://ftp.hs . uni-hamburg.de/pub/outgolng/FTS-Atlas

Caccin, B., Donati-Falchi, A., \& Falciani, R. 1976, Sol. Phys., 46, 29

Caccin, B., Falciani, R., \& Donati Falchi, A. 1978, Sol. Phys., 57, 13

Gustafsson, B., Meléndez, J., Asplund, M., \& Yong, D. 2010, Ap\&SS, 36

Hale, G. E., \& Adams, W. S. 1907, ApJ, 25, 300

Hastings, C. H. 1873, Nature, 8, 77

Livingston, W., Wallace, L., White, O. R., \& Giampapa, M. S. 2007, ApJ, 657, 1137

Lodders, K. 2003, ApJ, 591, 1220

Meléndez, J., Asplund, M., Gustafsson, B., \& Yong, D. 2009, ApJ, 704, L66

Pereira, T. M. D., Kiselman, D., \& Asplund, M. 2009, A\&A, 507, 417

Ramírez, I., Meléndez, J., \& Asplund, M. 2009, A\&A, 508, L17

Rodríguez Hidalgo, I., Collados, M., \& Vazquez, M. 1994, A\&A, 283, 263

Scharmer, G. B., Brown, D. S., Pettersson, L., \& Rehn, J. 1985, Appl. Opt., 24, 2558

Scharmer, G. B., Bjelksjö, K., Korhonen, T. K., Lindberg, B., \& Pettersson, B. 2003, in Innovative Telescopes and Instrumentation for Solar Astrophysics, ed. S. Keil, \& S. Avakyan, Proc. SPIE, 4853, 341

Vitas, N., Viticchiè, B., Rutten, R. J., \& Vögler, A. 2009, A\&A, 499, 301 